\title{
miR-1273g-3p promotes proliferation, migration and invasion of LoVo cells via cannabinoid receptor 1 through activation of ERBB4/PIK3R3/mTOR/S6K2 signaling pathway
}

\author{
MIN LI ${ }^{1}$, XIAOPING QIAN ${ }^{2}$, MINGZHEN ZHU ${ }^{3}$, AIYI LI ${ }^{3}$, MINGZHI FANG ${ }^{1}$, YONG ZHU ${ }^{4}$ and JINGYU ZHANG ${ }^{3}$ \\ ${ }^{1}$ Department of Oncology, The Third Affiliated Hospital of Nanjing University of Chinese Medicine, \\ Nanjing, Jiangsu 210000; ${ }^{2}$ Department of The Comprehensive Cancer Center, Affiliated Nanjing Drum Tower Hospital, \\ Nanjing University, Nanjing, Jiangsu 210008; ${ }^{3}$ The Department of Tumor-Chemotherapy, The Second People's Hospital of \\ Lianyungang, Lianyungang, Jiangsu 222023; ${ }^{4}$ National Medical Centre of Colorectal Disease, \\ The Third Affiliated Hospital of Nanjing University of Chinese Medicine, Nanjing, Jiangsu 210000, P.R. China
}

Received September 6, 2017; Accepted November 24, 2017

DOI: $10.3892 / \mathrm{mmr} .2018 .8397$

\begin{abstract}
MicroRNAs (miR) are important in various crucial cell processes including proliferation, migration and invasion. Dysregulation of miRNAs have been increasingly reported to contribute to colorectal cancer. However, the detailed biological function and potential mechanisms of miR-1273g-3p in colorectal cancer remain poorly understood. The expression levels of miR-1273g-3p in human colorectal cancer LoVo cell lines were detected via reverse transcription-quantitative polymerase chain reaction (RT-qPCR). The target genes of miR-1273g-3p were predicted by bioinformatics and verified by a luciferase reporter assay, RT-qPCR and western blotting. The MTT, wound-healing and Transwell assays were used to examine the biological functions of miR-1273g-3p in LoVo cells. The potential molecular mechanisms of miR-1273g-3p on LoVo cell proliferation, migration and invasion was detected by western blotting. The results of the present study demonstrated that miR-1273g-3p expression was extensively upregulated in LoVo cells compared with the normal colon epithelial NCM460 cell line. Further studies indicated that miR-1273g-3p inhibitor significantly suppressed LoVo cell proliferation, migration and invasion compared with inhibitor
\end{abstract}

Correspondence to: Dr Jingyu Zhang, The Department of Tumor-Chemotherapy, The Second People's Hospital of Lianyungang, 161 Xingfu Road, Haizhou, Lianyungang, Jiangsu 222023, P.R. China E-mail: zhangjingyu0018@163.com

Dr Yong Zhu, National Medical Centre of Colorectal Disease, The Third Affiliated Hospital of Nanjing University of Chinese Medicine, 1 Jinling Road, Nanjing, Jiangsu 210000, P.R. China E-mail: yangyong8100@163.com

Keywords: miR-1273g-3p, cannabinoid receptor 1, colorectal cancer, proliferation, migration, invasion, ERBB4/PIK3R3/mTOR/S6K2 pathway control. Following this, the cannabinoid receptor 1 (CNR1) was identified as a direct target gene of miR-1273g-3p. Knockdown of CNR1 restored the phenotypes of LoVo cells transfected with miR-1273g-3p inhibitor. Furthermore, the potential molecular mechanism of miR-1273g-3p on LoVo cell proliferation, migration and invasion may be mediated by activating the Erb-B2 receptor tyrosine kinase 4 (ERBB4)/phosphoinositide-3-kinase regulatory subunit 3 (PIK3R3)/mechanistic target of rapamycin (mTOR)/S6 kinase 2 (S6K2) signaling pathway. These observations indicated that $\mathrm{miR}-1273 \mathrm{~g}-3 \mathrm{p}$ promoted the proliferation, migration and invasion of LoVo cells via CNR1, and this may have occurred through activation of the ERBB4/PIK3R3/mTOR/S6K2 signaling pathway, suggesting that $\mathrm{miR}-1273 \mathrm{~g}-3 \mathrm{p}$ may serve as a novel therapeutic target for the effective treatment of colorectal cancer.

\section{Introduction}

Colorectal cancer (CRC) is a common gastrointestinal malignancy and is the third-leading cause of cancer death in the world (1). Molecular pathogenesis of CRC is complicated and poorly understood. Several risk factors are associated with CRC progression, including aging, genetic aberrations, and chronic intestinal inflammation (2). Although CRC-related oncogenic factors have been extensively studied, the underlying mechanisms remains to be elucidated.

MicroRNAs (miRNA), 19-22 nucleotide non-coding RNA molecules, have been identified as key negative regulators of gene expression by binding to the 3'-untranslated regions (UTR) of the target mRNAs of protein-coding genes, resulting in mRNA cleavage or the inhibition of mRNA translation (3). Accumulating evidence suggested that miRNAs regulate the expression of genes involved in several important cancer-related processes including cell adhesion, proliferation, apoptosis and tumorigenesis, including colorectal cancer (2). Zhang et al has reported that miR-520a-3p could suppressed colorectal cancer cell migration via the regulation of EGFR expression (4). Song et al has reported miR-582 could enhanced 
the proliferation and migration ability of the CRC cells by decreasing PTEN expression (5). Exploration of deregulated miRNAs in carcinogenesis and metastasis of CRC, may reveal novel therapeutic targets for the effective treatment of CRC (6).

miR-1273g-3p, a length of $21 \mathrm{nt}$ non-coding RNA molecule, is coded in an intron of the SCP2 gene, and was first discovered in 2011 years (7). Previous study has reported that miR-1273g-3p participates in acute glucose fluctuation-induced proliferation attenuation in human umbilical vein endothelial cells (8). And miR-1273g-3p could modulate activation and apoptosis of hepatic stellate cells by directly targeting PTEN in HCV-related liver fibrosis (9). In addition, recent study has identified that miR-1273g-3p is upregulated in lung cancer A549 cells and promotes A549 cells migration by targeting CNR1 (10). However, the expression levels and biological roles of miR-1273g-3p in CRC and underlying mechanism for its functional remains not fully elucidated.

Cannabinoid receptor 1 (CNR1), the gene encoding cannabinoid receptors (CB1), a member of the endocannabinoid system, is highly expressed in the central and peripheral nervous systems (PNS) in mammalians $(11,12)$. CNR1 expression outside the brain has been associated with cell migration and tumor procession (1). For example, PAX3-FOXO1 induces Cannabinoid receptor 1 to enhance cell invasion and metastasis (13). In addition, CNR1 is coupled to several signaling pathways directly involved in cell survival, migration, proliferation and apoptosis, including MAPK, cyclic AMP, and PI3K-Akt pathways $(14,15)$.

In the present study, we identified miR-1273g-3p was frequently up-regulated in CRC cell lines LoVo. We confirmed that miR-1273g-3p works as a tumor initiator during proliferation, migration and invasion of LoVo cells. Moreover, CNR1 was identified as direct and functional target of miR-1273g-3p. Furthermore, our results showed that miR-1273g-3p promotes proliferation, migration and invasion of LoVo cells via CNR1 may be through the activation of ERBB4/PIK3R3/mTOR/S6K2 signaling pathway.

\section{Materials and methods}

Cell culture. Human colorectal cancer cell line LoVo, obtained from American Type Culture Collection (Manassas, VA, USA), was cultured in dulbecco modified Eagle medium (DMEM; Sigma-Aldrich; Merck KGaA, Darmstadt, Germany) containing high glucose, supplemented with $10 \%$ fetal bovine serum (FBS; Gibco; Thermo Fisher Scientific, Inc., Waltham, MA, USA), $2 \mathrm{mM}$ L-glutamine, $100 \mathrm{U} / \mathrm{ml}$ penicillin, and $100 \mu \mathrm{g} / \mathrm{ml}$ streptomycin (P/S; Invitrogen; Thermo Fisher Scientific, Inc.) and incubated in a $5 \% \mathrm{CO}_{2}$ high-humidity atmosphere at $37^{\circ} \mathrm{C}$ with saturated humidity. The normal colon epithelial NCM460 cell was cultured at the same condition.

Oligonucleotide transfection. miR-1273g-3p mimic, miR-1273g-3p inhibitor (anti-miR-1273g-3p, chemically modified antisense oligonucleotides designed to target specifically against mature miR-1273g-3p) and their corresponding controls (mimic control and inhibitor control) were purchased from Guangzhou RiboBio Co., Ltd., (miR20022742-1-5; Guangzhou, China). CNR1siRNA was synthesized Shanghai GenePharma Co., Ltd., (Shanghai, China). When the LoVo cells was grown to $70-80 \%$ confluence. miR-1273g-3p mimic or inhibitor, CNR1siRNA was transfected into cell using Lipofectamine 2000 reagents (Invitrogen; Thermo Fisher Scientific, Inc.) according to the manufacturer's protocol. The culture media was replaced with fresh medium $4 \mathrm{~h}$ later.

MTT assay. The influence of miR-1273g-3p on LoVo cell proliferation was tested using the MTT (3-(4, 5-dimethyl-2thiazolyl)-2, 5-diphenyl-2-H-tetrazolium bromide) (Sigma-Aldrich; Merck KGaA) assay. LoVo cells were seeded in 96-well plates at a density of $5 \times 10^{3}$ per well and cultured at $37^{\circ} \mathrm{C}$ in an atmosphere of $5 \% \mathrm{CO}_{2}$ for $24 \mathrm{~h}$. According to Lipofectamin $^{\mathrm{TM}} 2000$ manual, LoVo cells were transfected with miR-1273g-3p inhibitor, inhibitor control, or co-transfected with miR-1273g-3p inhibitor and CNR1 siRNA for 24, 48 or $72 \mathrm{~h}$. Then adding $20 \mu \mathrm{l} 5 \mathrm{mg} / \mathrm{ml}$ MTT solution to each well and incubation for $4 \mathrm{~h}$ at $37^{\circ} \mathrm{C}$. The medium was removed and $150 \mu \mathrm{l}$ dimethyl sulfoxide was added to each well, the absorbance was measured at $570 \mathrm{~nm}$ on a microplate reader (Multiskan Spectrum; Thermo Fisher Scientific, Inc.). Each assay was performed with 5 replicate wells for each condition.

Wound-healing assays. For cell migration assay, LoVo cells were seeded at $1.5 \times 10^{5}$ per well into 6 well plates in complete medium, and cultured at $37^{\circ} \mathrm{C}$ in an atmosphere of $5 \% \mathrm{CO}_{2}$ for $24 \mathrm{~h}$. According to Lipofectamin ${ }^{\mathrm{TM}} 2000$ manual, LoVo cells were transfected with miR-1273g-3p inhibitor, inhibitor control, or co-transfected with miR-1273g-3p inhibitor and CNR1 siRNA. At $24 \mathrm{~h}$ after transfection, a linear wound was carefully made by a $20 \mu 1$ sterile pipette tip across the confluent cell monolayer to leave a scratch of approximately $0.4-0.5 \mathrm{~mm}$ in width, and the cell debris was removed by washing with phosphate-buffered saline and incubated with a fresh serum free culture medium. The wounded monolayers were then photographed at $0,24 \mathrm{~h}$ after wounding.

Cell invasion assay. For cell invasion assay, matrigel coated transwells (BD Biosciences, Franklin, NJ, USA) containing $8 \mu \mathrm{m}$ pores was used (BD Biosciences, Mountain View, CA, USA). LoVo cells were transfected with miR-1273g-3p inhibitor, inhibitor control, or co-transfected with miR-1273g-3p inhibitor and CNR1 siRNA. The transfected cells were seeded into the upper chamber at $5 \times 10^{4}$ cells per well. DMEM supplemented with containing $10 \%$ FBS was added to the bottom chambers. LoVo cells was allowed to incubate for $24 \mathrm{~h}$ at $37^{\circ} \mathrm{C}$. After the incubation period, the filter was removed, and non-invasion cells on the upper side of the filter were detached using a cotton swab. Then the filter was fixed in $4 \%$ formaldehyde. Cell were stained with $0.1 \%$ crystal violet for $20 \mathrm{~min}$ and counted from three random fields.

Western blot analysis. After transfection for $24 \mathrm{~h}$, total proteins were extracted from the LoVo cells with radio immunoprecipitation assay (RIPA) lysis buffer (Pierce; Thermo Fisher Scientific, Inc.). Protein samples were quantified with the Pierce BCA Protein assay kit (Pierce; Thermo Fisher Scientific, Inc.) The total protein $(80 \mu \mathrm{g} /$ well $)$ from each sample was separated by 6 and $8 \%$ sodium dodecyl sulfate-polyacrylamide gelelectrophoresis (SDS/PAGE) and transferred to a polyvinylidene difluoride (PVDF) membrane (EMD Millipore, Billerica, 
MA, USA). After blocking, the PVDF membranes were incubated with the following primary antibodies: ERBB4 (cat. no. AP7631a; dilution, 1:1,000; Abgent, Inc., San Diego, CA, USA), S6K2 (cat. no. AP8009c; dilution, 1:1,000; Abgent, Inc.), PIK3R3 (cat. no. AP8025a; dilution, 1:1,000; Abgent, Inc.), mTOR (cat. no. S2481; dilution, 1:1,000; Abgent, Inc.), and GAPDH (cat. no. 97166; dilution, 1:1,000; Santa Cruz Biotechnology, Inc., Dallas, TX, USA) overnight at $4^{\circ} \mathrm{C}$, then incubated with horseradish peroxidase-conjugated anti-mouse IgG (cat. no. A9044; dilution, 1:2,500; Sigma-Aldrich; Merck $\mathrm{KGaA}$ ) and horse radish peroxidase-conjugated anti-rabbit IgG (cat. no. A0545; dilution, 1:2,500; Sigma-Aldrich; Merck $\mathrm{KGaA}$ ) as the secondary antibodies for $1 \mathrm{~h}$ at room temperature. The immunoreactive bands were visualized with an enhanced chemiluminescence kit (20158; Thermo Fisher Scientific, Inc.). Images were captured with a Fuji LAS3000-mini imaging system (Fujifilm, Tokyo, Japan), and immunoreactive bands were quantified.

Reverse transcription-quantitative polymerase chain reaction $(R T-q P C R)$. Total RNA was extracted using TRIzol reagent (Invitrogen; Thermo Fisher Scientific, Inc.) according to the manufacture's protocol. First-strand cDNA was synthesized with the PrimeScript RT reagent kit (Roche Applied Science, Pleasanton, CA, USA). qRT-PCR analyse was carried out to detect mRNA expression using SYBR Premix Ex Taq (ROX; Roche Applied Science). GAPDH mRNA levels was used as a reference control. The primer sequences are as follows: CNR1 5'-TCCACTTCTTTTCCGCCTCC-3' (forward), CNR1 5'-AATCTCTTTGCCCCTTCGCA-3' (reverse); ERBB4: 5'-CCAAGAGGACAGTAGCACCC-3' (forward), ERBB4:5'-CTGGATTCAGGTATTCTTGTTTGGG-3'(reverse); PIK3R3: 5'-CTTGCTCTGTGGTGGCCGAT-3' (forward), PIK3R3: 5'-GACGTTGAGGGAGTCGTTGT-3' (reverse); mTOR: 5'-ATGCAGCTGTCCTGGTTCTC-3' (forward), mTOR: 5'-AATCAGACAGGCACGAAGGG-3' (reverse); S6K2: 5'-TGA CTCACAGCAGCAAGATGT-3' (forward), S6K2: 5'-AGTACT CCCACAGCCAGGAA-3' (reverse); GAPDH 5'-ACCACAGTC CATGCCATCAC-3' (forward), GAPDH 5'TCACCACCCTGT TGCTGTA-3' (reverse).

To quantify the expression of miR-1273g-3p, qRT-PCR was performed using TaqMan microRNA assays (Applied Biosystems; Thermo Fisher Scientific, Inc.) and U6 snRNA was used as an endogenous control for miR-1273g-3p. The thermocycling parameters were $95^{\circ} \mathrm{C}$ for $10 \mathrm{~min}$, followed by 40 cycles of $95^{\circ} \mathrm{C}$ for $10 \mathrm{sec}$ and by $60^{\circ} \mathrm{C}$ for $30 \mathrm{sec}$. The relative expression level of $\mathrm{CNR} 1$ and miR-1273g-3p were cultured using the comparative delta $\mathrm{Cq}\left(2^{-\Delta \Delta \mathrm{Cq}}\right)$ method (16). Each sample was analyzed in triplicate.

Luciferase reporter assay. To construct CNR1 3'UTR plasmid, the full-length 3'UTR of human CNR1 mRNA containing the putative miR-1273g-3p binding sequence (CNR1 3'-UTR-WT) was cloned into the pGL3 promotor vector (Promega Corporation, Madison, WI, USA). Mutant-type CNR13'-UTR (CNR1 3'-UTR-MUT) was amplifed using site-directed mutagenesis kit (Enzynomic, Daejeon, Korea), with CNR1 3'-UTR-WT as a template. For the dual luciferase assay, LoVo cells were cultured in 24-well plates ( $5 \times 10^{4}$ cells per well) for $24 \mathrm{~h}$ and co-transfected with $100 \mathrm{ng}$ of CNR1-3'UTR-WT or CNR1-3'UTR MUT and $50 \mathrm{nM}$ of miR-1273g-3p mimics or mimics control, using Lipofectamine 2000 transfection reagent (Invitrogen; Thermo Fisher Scientific, Inc.), according to the manufacturer's protocol. After $6 \mathrm{~h}$, the Opti-MEM (Invitrogen; Thermo Fisher Scientific, Inc.) transfection medium was replaced with DMEM supplemented with $10 \%$ FBS. Cells were harvested and assayed with the Luciferase Assay System (Promega Corporation) at $24 \mathrm{~h}$ after transfection. The Renilla luciferase activity was normalized to the firefly luciferase activity. The experiment was conducted in triplicate.

Statistical analysis. Statistical analysis were performed using SPSS v17.0 software (SPSS, Inc., Chicago, IL, USA). Quantitative variables were presented as means \pm SEM. Data were analyzed by one-way analysis of variance followed with the Schefffe post hoc test. ${ }^{*},{ }^{* *},{ }^{\# \#},{ }^{* * *}$ manifested $\mathrm{P}<0.05, \mathrm{P}<0.01$, $\mathrm{P}<0.001$, respectively. $\mathrm{P}<0.05$ was considered to indicate a statistically significant difference.

\section{Results}

LoVo cells miR-1273g-3p is dramatically upregulated in LoVo cells. To assess the role of miR-1273g-3p in colorectal cancer, the expression of miR-1273g-3p in human colorectal cancer LoVo cells was examined. qRT-PCR analysis showed that the levels of miR-1273g-3p was markedly upregulated in the LoVo cells compared with the normal colon epithelial NCM460 cell (Fig. 1). The results indicated that miR-1273g-3p may be involved in the malignant progression of colorectal cancer.

CNR1 is a direct target gene of miR-1273g-3p in LoVo cells. Firstly, the targets of miR-1273g-3p were analyzed using miRecords resource from three independent databases: PicTar (http://pictar.mdc-berlin.de/), TargetScan (http://www.targetscan.org/vert_71/), and miRBase (http://www.mirbase.org/search.shtml). CNR1 is predicted a theoretical target gene of miR-1273g-3p (Table I). To obtain direct evidence that CNR1 is a direct target of miR-1273g-3p, we characterized the binding site of $\mathrm{miR}-1273 \mathrm{~g}-3 \mathrm{p}$ in the $3^{\prime}$ UTR of CNR1 mRNA (Fig. 2A). In a dual luciferase reporter assay, miR-1273g-3p mimic was transfected in LoVo cells causing the CNR1-3'-UTR-dependent luciferase activity significantly decreased but did not affect the luciferase activity of the mutant reporter compared with the mimic control (Fig. 2B). Meanwhile, qRT-PCR and western blot analyses also confirmed this prediction. The results showed that post LoVo cells transfection with miR-1273g-3p inhibitor, the CNR1 mRNA (Fig. 2C) and protein expression (Fig. 2D) level were significantly upregulated, higher than that before transfection. Collectively, these data confirmed that CNR1 as a direct target gene of miR-1273g-3p in LoVo cells.

miR-1273g-3p promotes the proliferation, migration and invasion of LoVo cells via directly targeting CNR1. To further investigate the effect of miR-1273g-3p on colon cancer cells, and whether this effect is mediated by CNR1 gene. LoVo cells were transfected with miR-1273g-3p inhibitor, inhibitor control or co-transfected with miR-1273g-3p inhibitor and CNR1 siRNA. Cells were harvested after $24 \mathrm{~h}$ and successful 


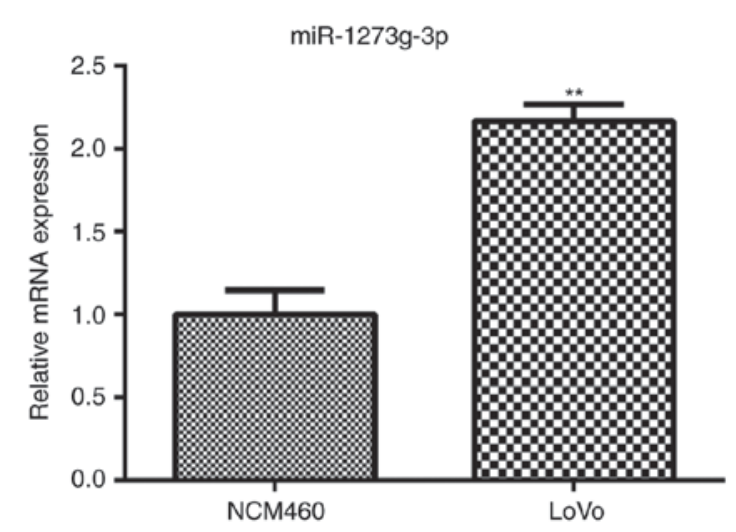

Figure 1. miR-1273g-3p is dramatically upregulated in LoVo cells. miR-1273g-3p expression in human colorectal cancer LoVo cells and normal colon epithelial NCM460 cell was examined by qRT-PCR. Data are expressed as mean \pm standard error. ${ }^{* *} \mathrm{P}<0.01$ vs. NCM460 group. miR, microRNA.

transfection and subsequent downregulation of the expression of miR-1273g-3p was confirmed by qRT-PCR (Fig. 3A).

Transfected with CNR1 small interfering RNA (siRNA) in LoVo cells which resulted in a $>90 \%$ reduction in the levels of CNR1 protein (Fig. 3B) and mRNA (Fig. 3C). MTT assay revealed that miR-1273g-3p inhibitor significantly inhibited the growth of LoVo cells compared with the control group (Fig. 3D). In addition, miR-1273g-3p inhibitor significantly inhibited LoVo cells migration and invasion compared with the control group in wound-healing assays (Fig. 3E) and cell invasion assay (Fig. 3F-G). Remarkably, the resulting constitutively expressed CNR1siRNA abrogated the previously reduced proliferation (Fig. 3D), migration (Fig. 3E) and invasion (Fig. 3F-G) initiated by miR-1273g-3p inhibitor. Taken together, these results proved that miR-1273g-3p promotes LoVo cell proliferation, migration and invasion via directly targeting CNR1.

miR-1273g-3p increases ERBB4/PIK3R3/mTOR/S6K2 expression via directly targeting $C N R 1$. It has been reported that CNR1 is coupled to several signaling pathways directly involved in cell migration and tumor growth, including cyclic AMP, and PI3K-Akt pathways $(17,18)$. Until now, ERBB4, S6K2, PIK3R3 and mTOR have been plays a critical roles in cell movement, growth and development (6). To clarify the underlying molecular mechanisms of miR-1273g-3p on LoVo cells proliferation, migration and invasion. We then detected ERBB4, PIK3R3, mTOR, and S6K2 expression in LoVo cells were transfected with miR-1273g-3p inhibitor, inhibitor control, or co-transfected with miR-1273g-3p inhibitor and CNR1siRNA by western blot and qRT-PCR. As shown in Fig. 4, the results revealed that silencing of CNR1 could significantly upregulated the protein (Fig. 4A) and mRNA (Fig. 4B-E) levels of ERBB4, PIK3R3, mTOR and S6K2 in LoVo cells. Furthermore, inhibition of miR-1273g-3p significantly decreased the protein (Fig. 4A) and mRNA (Fig. 4B-E) levels of ERBB4, PIK3R3, mTOR and S6K2 compare with the control group in LoVo cells, which could be revised by CNR1siRNA. Taken together, these data indicated that miR-1273g-3p promotes proliferation, migration and invasion of LoVo cells via CNR1 may be through the activation of ERBB4, PIK3R3, mTOR, and S6K2 pathway.
Table I. Potential targets mRNA of miR-1273g-3p.

\begin{tabular}{lll}
\hline miRNA & Target gene & \multicolumn{1}{c}{ Gene ID } \\
\hline miR-1273g-3p & CNR1 & NM_001160226 \\
& PTEN & NM_000314 \\
MDM4 & NM_002393 \\
NOL9 & NM_024654 \\
& PLCXD1 & NM_018390 \\
& SAR1B & NM_016103 \\
& ZNF850 & NM_001193552 \\
& CYP20A1 & NM_020674 \\
\hline
\end{tabular}

\section{Discussion}

Increasing evidence suggests that miRNAs have important roles in carcinogenesis and metastasis (19). Dysregulation of miRNAs is critically involved in the development and progression of colorectal cancer (20). The present study first validated that the expression of miR-1273g-3p was significantly upregulated in LoVo cells by RT-PCR. In addition, miR-1273g-3p promoted LoVo cells proliferation, migration and invasion. The results indicated that miR-1273g-3p may act as a tumor initiator and play an accelerating role in the progression of CRC. However, the potential mechanisms of miR-1273g-3p in human colorectal cancer LoVo cells remains unclear.

It has been reported that miR-1273g-3p has 1,330 binding sites on 1,074 mRNAs (7). According to the prediction software, we list some potential targets mRNA of miR-1273g-3p in Table I. Previous study has indicated that miR-1273g-3p modulates activation and apoptosis of hepatic stellate cells by directly targeting PTEN in HCV-related liver fibrosis (9). In addition, recent study has identified that miR-1273g-3p is upregulated in lung cancer A549 cells and promotes A549 cells migration by targeting CNR1 (10). CNR1, the gene encoding cannabinoid receptors 1 (CB1), is highly expressed in the central and peripheral nervous systems (PNS) in mammalians and is involved in neuromodulatory functions (21). CNR1 expression outside the brain has been associated with cell migration and tumor growth (12). Previous studies demonstrated that CNR1 may provide a therapeutic target in tumor intervention and its anti-tumor actions by proliferation arrest or induction of apoptosis, suppression of angiogenesis and tumor metastatic spreading $(18,22)$. Recently, several studies have also suggested that CNR1 plays a role in cell migration using cell lines, although the results remain controversial $(15,23)$. It has been reported that blocking of the CB1 receptor, rather than its stimulation could inhibited lung metastasis formation (13). By contrast, loss of the CB1 receptor in a genetic model of colon cancer induced tumor growth (24). Our findings demonstrated that as a target gene of miR-1273g-3p, CNR1 may be negatively regulated by miR-1273g-3p at the transcriptional level via binding of the 3'UTR of CNR1 mRNA in LoVo cells. Furthermore, our results revealed that CNR 1 downregulation was necessary to mediate the effects of miR-1273g-3p on cellular 

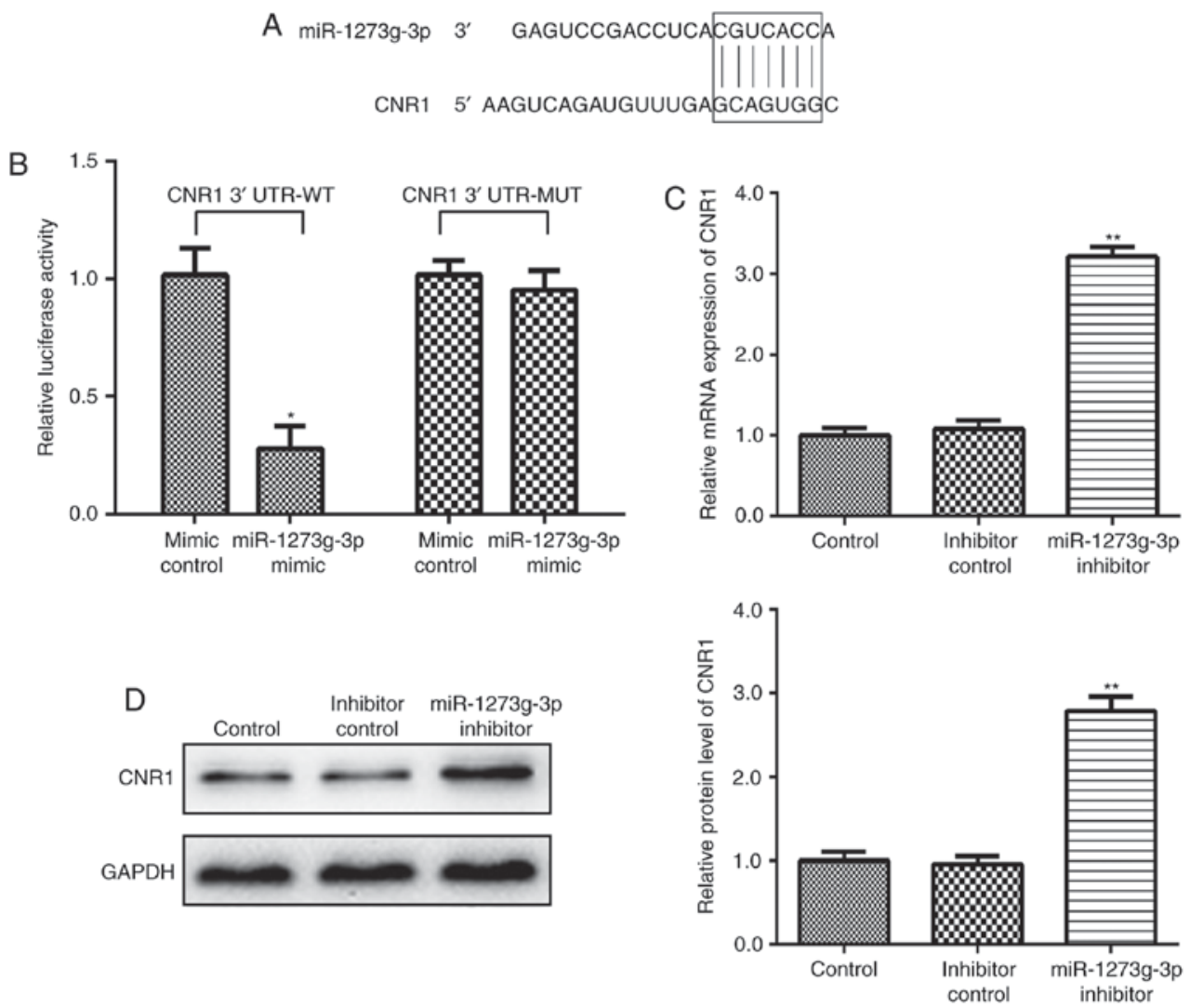

Figure 2. CNR1 is a target of miR-1273g-3p in LoVo cells. (A) Sequence of the miR-1273g-3p binding site within the human CNR1 3'-UTR and schematic diagram of the reporter construct showing the wild type (WT) of CNR1 3'-UTR sequence. (B) Luciferase activity in cells co-transfected with CNR1 3'UTR WT/MUT and miR-1273g-3p mimic/mimic control. (C) mRNA expression levels of CNR1 in LoVo cells transfected with miR-1273g-3p inhibitor or inhibitor control was assessed by qRT-PCR. (D) Protein expression levels of CNR1 in LoVo cells transfected with $1273 \mathrm{~g}-3 \mathrm{p}$ inhibitor or inhibitor control was detected by western blot. Data are expressed as mean \pm standard error. ${ }^{*} \mathrm{P}<0.05,{ }^{* *} \mathrm{P}<0.01$ vs. control. CNR1, cannabinoid receptor 1 ; miR, microRNA; UTR, untranslated region; WT, wildtype; MUT, mutated.

proliferation, migration and invasion. Taken together, our data clearly indicated that CNR1 was a direct target of miR-1273g-3p in LoVo cells.

Recent studies have suggested that cell migratory responses were often mediated by Gi protein-coupled receptors $(25,26)$. It has been reported that CNR1 is coupled to pertussis toxin-sensitive $\mathrm{G}$ proteins and regulated several signaling pathways directly involved in cell survival, migration, proliferation and apoptosis, including MAPK, cyclic AMP, and PI3K-Akt pathways $(27,28)$. Recent study has indicated that miR-1273g-3p suppresses PTEN expression and negatively regulates the AKT pathway in LX-2 cells (9). However, the potential molecular mechanism of miR-1273g-3p promoting LoVo cells migration via CNR1 was poorly understood. Until now, ERBB4, S6K2, PIK3R3 and mTOR have been plays critical roles in cell movement, growth and development. ERBB4, S6K2 (P70S6Kb), PIK3R 3 and mTOR, potential metastasis promoters, were identified as direct and functional targets of miR-193a-3p/5p. ERBB4 and S6K2 were the direct targets of miR-193a-3p and that PIK3R3 and mTOR were the direct targets of miR-193a-5p in NSCLC. Therefore, miR-193a-3p/5p could inhibit the metastasis of NSCLC by interacting with the ERBB signaling pathway (6). ERBB4 activated the PI3K-AKT cascade contribute to the progression of pancreatic cancer (29). mTOR and its downstream effector S6K2 were frequently upregulated in breast cancer, and assumed to be driving forces in tumourigenesis (30). PIK3R3/AKT pathway was involved in the tumorigenesis and progression of gastric cancer (31). Inhibition of PI3K/mTOR pathway impaired breast tumor invasion and metastasis (32). The present study indicated that cannabinoid receptor 1 (CNR1) was identified as a direct target gene of miR-1273g-3p. Whether ERBB4/PIK3R3/mTOR/S6K2 signaling pathway was involved in the effect of miR-1273g-3p on LoVo cells remains unknown. In the present study, our results showed that transfection of miR-1273g-3p inhibitor significantly downregulated the proteins and mRNA levels of ERBB4, PIK3R3, mTOR and S6K2 in LoVo cells, which could be reversed by CNR1siRNA. These results suggested that the dysregulation of the ERBB4/PIK3R3/mTOR/S6K2 signaling pathway may be a potential mechanisms of miR-1273g-3p promoting the proliferation, migration and invasion of LoVo cells.

In conclusion, our results showed that miR-1273g-3p was frequently upregulated in LoVo cells. The overexpression of miR-1273g-3p promoted LoVo cells proliferation, migration and invasion via directly targeting CNR1, and the potential mechanisms may be through the activation of ERBB4/PIK3R3/mTOR/S6K2 signaling pathway. Importantly, these findings indicated that miR-1273g-3p may represent a novel target for therapeutic intervention to CRC. However, 

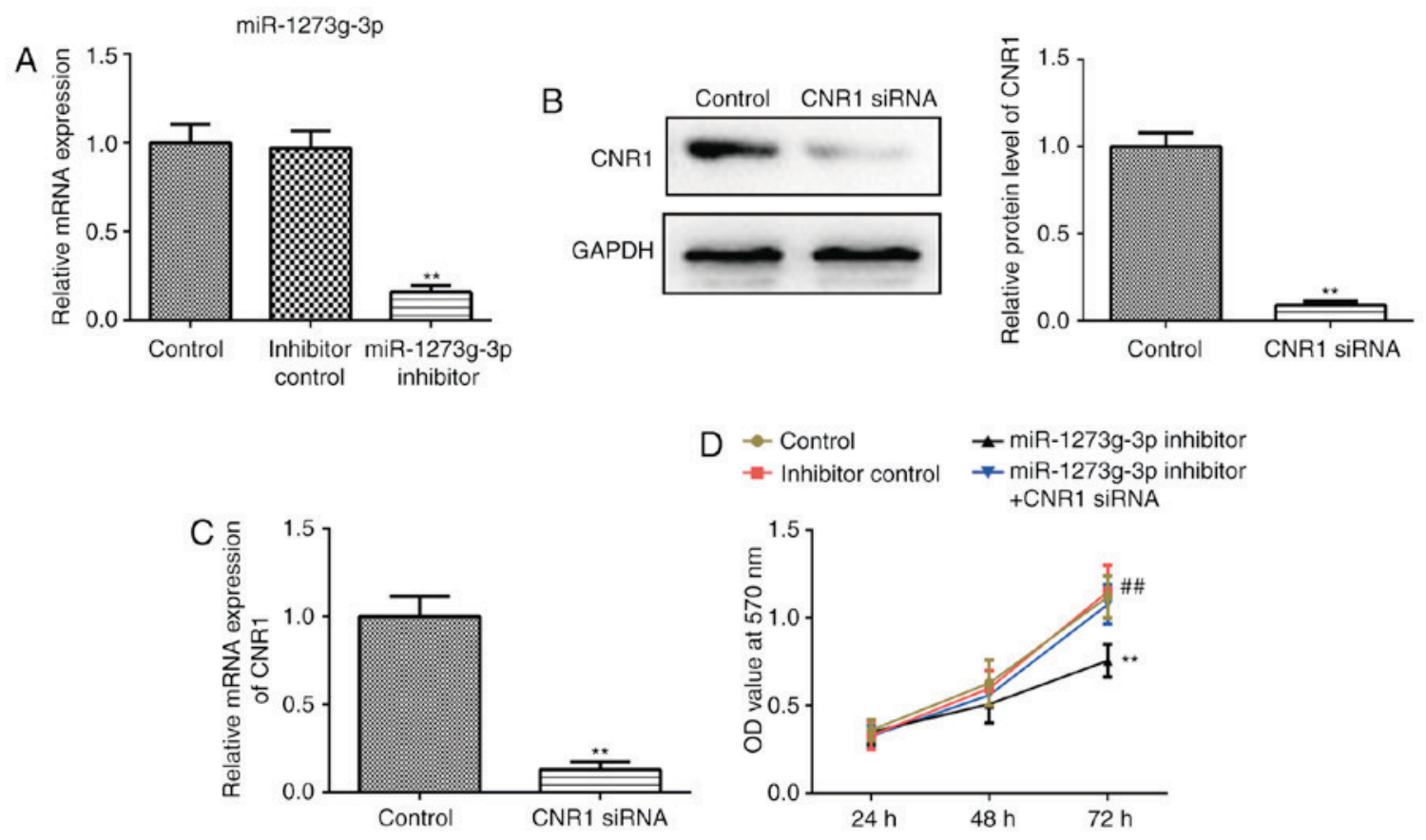

E

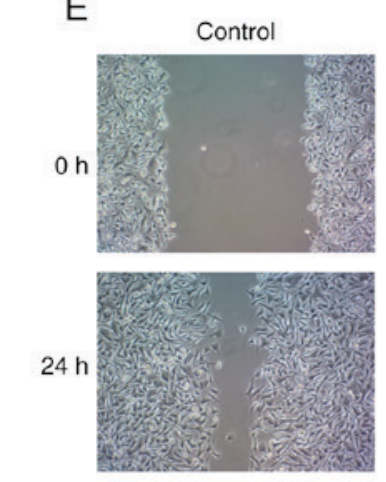

Inhibitor control
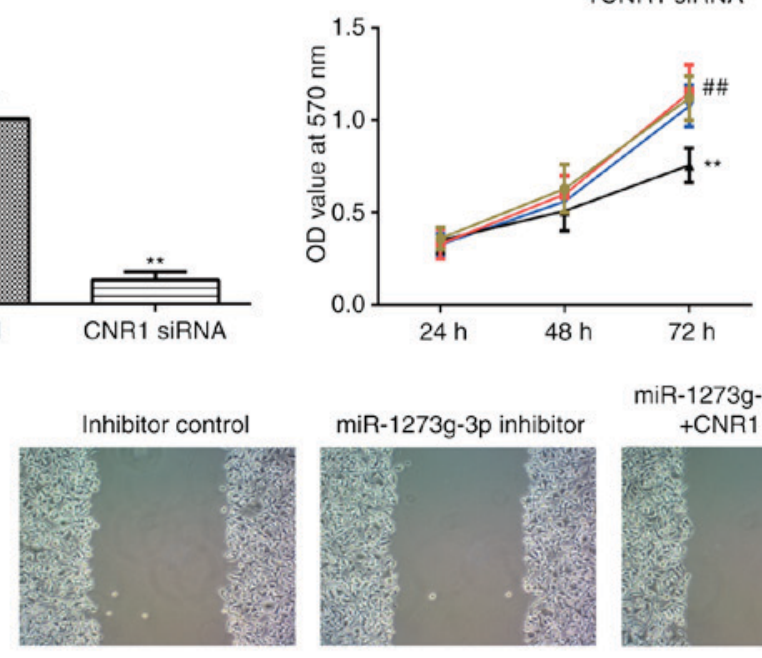

miR-1273g-3p inhibitor

miR-1273g-3p inhibitor
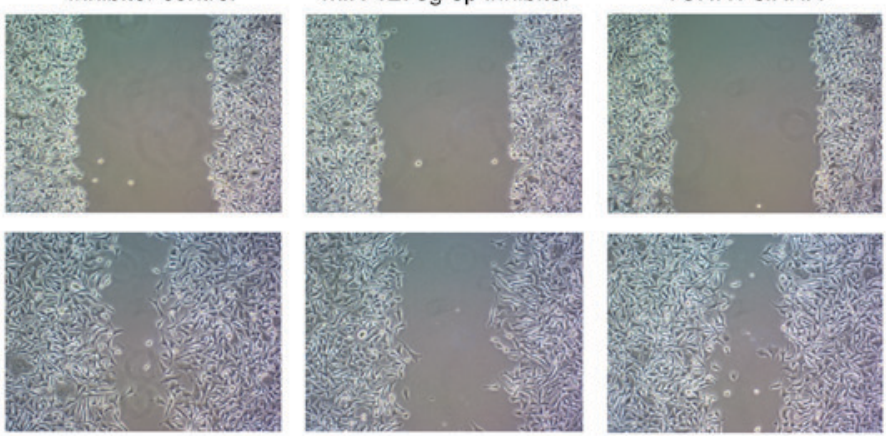

$\mathrm{F}$

Inhibitor control

miR-1273g-3p inhibitor

miR-1273g-3p inhibitor
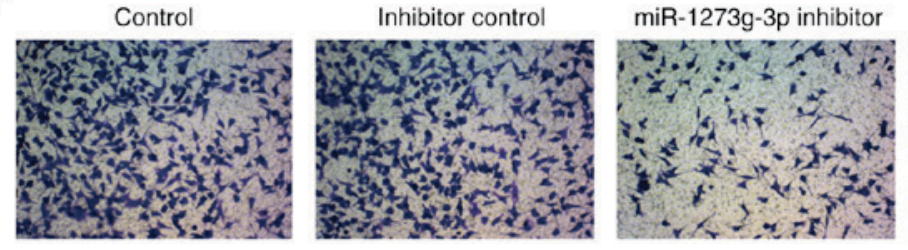

+CNR1 SiRNA

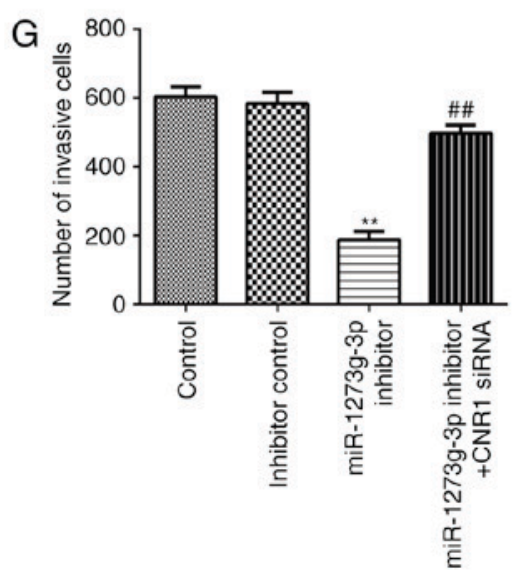

Figure 3. miR-1273g-3p promotes the proliferation, migration and invasion of LoVo cells via directly targeting CNR1. (A) qRT-PCR was used to examine the miR-1273g-3p expression in LoVo cells transfected with miR-1273g-3p inhibitor or inhibitor control. (B) Western blot and (C) qRT-PCR were used to examine the protein and mRNA expression levels of CNR1 in LoVo cells transfected with CNR1siRNA. (D) MTT, (E) Wound-healing assays and (F) Cell invasion assay were used to examine cell proliferation, migration and invasion of LoVo cells transfected with miR-1273g-3p inhibitor or co-transfected miR-1273g-3p inhibitor and CNR1siRNA. (G) Quantitative analysis of the number of invasion cells. Data are expressed as mean \pm standard error. ${ }^{* *} \mathrm{P}<0.01 \mathrm{vs}$. control. ${ }^{\# \#} \mathrm{P}<0.01$ vs. miR-1273g-3p inhibitor. CNR1, cannabinoid receptor 1; OD, optical density; miR, microRNA. 

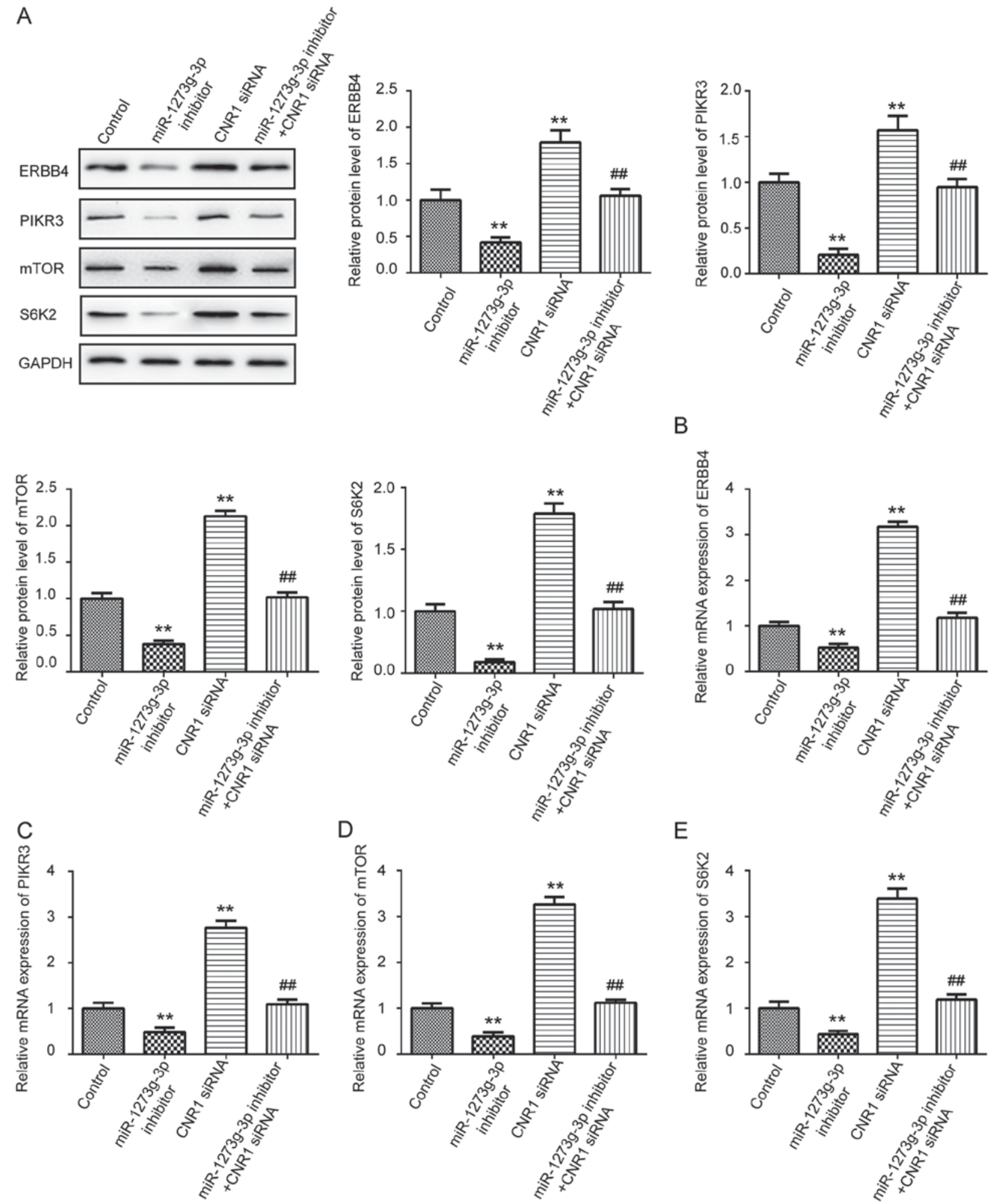

$\mathrm{E}$

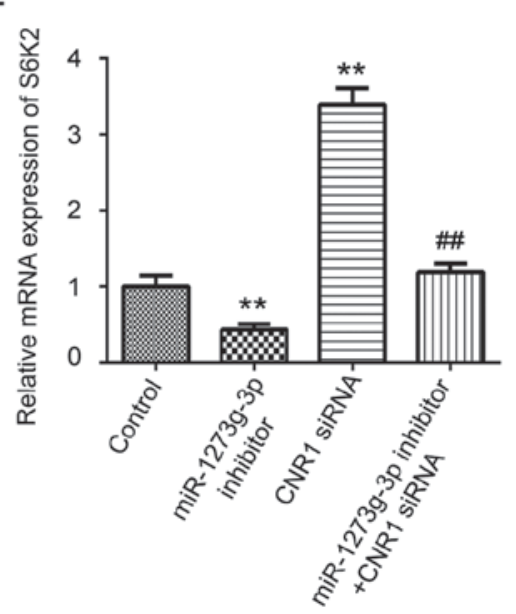

Figure 4. miR-1273g-3p increases ERBB4/PIK3R3/mTOR/S6K2 expression via directly targeting CNR1. (A) Western blot was used to examine the protein expression levels of ERBB4, PIK3R3, mTOR and S6K2 in LoVo cells transfected with miR-1273g-3p inhibitor, CNR1siRNA, or co-transfected with miR-1273g-3p inhibitor and CNR1siRNA. qRT-PCR was conducted to examine the mRNA expression of ERBB4 (B), PIK3R3 (C), mTOR (D) and S6K2 (E) in LoVo cells transfected with miR-1273g-3p inhibitor, CNR1siRNA, or co-transfected with miR-1273g-3p inhibitor and CNR1siRNA. Data are expressed as mean \pm standard error. ${ }^{* *} \mathrm{P}<0.01$ vs. control. ${ }^{\# \#} \mathrm{P}<0.01$ vs. miR-1273g-3p inhibitor. CNR1, cannabinoid receptor 1; miR, microRNA.

a limitation of this study is normal cells should always be included in these studies as manipulation of expression always has an effect and would like to keep it to the minimum for control cells. Further studies to investigate this possibility are ongoing in our laboratory.

\section{Acknowledgements}

The present study was supported by the department of Health projects of Lianyungang city (grant no: 201715) and clinic science and technology projects of Jiangsu collage (grant no: JLY20140133). 


\section{References}

1. Xie H, Sun X, Piao Y, Jegga AG, Handwerger S, Ko MS and Dey SK: Silencing or amplification of endocannabinoid signaling in blastocysts via CB1 compromises trophoblast cell migration. J Biol Chem 287: 32288-32297, 2012.

2. Wong MC, Ching JY, Chan VC, Lam TY, Luk AK, Wong SH, Ng SC, Ng SS, Wu JC, Chan FK and Sung JJ: Colorectal cancer screening based on age and gender: A cost-effectiveness analysis. Medicine (Baltimore) 95: e2739, 2016.

3. Bartel DP: MicroRNAs: Target recognition and regulatory functions. Cell 136: 215-233, 2009.

4. Zhang R, Liu R, Liu C, Niu Y, Zhang J, Guo B, Zhang CY, Li J, Yang $J$ and Chen $\mathrm{X}$ : A novel role for MiR-520a-3p in regulating EGFR expression in colorectal cancer. Cell Physiol Biochem 42: 1559-1574, 2017.

5. Song B, Long Y, Liu D, Zhang W and Liu C: MicroRNA-582 promotes tumorigenesis by targeting phosphatase and tensin homologue in colorectal cancer. Int $\mathbf{J}$ Mol Med 40: 867-874, 2017.

6. Yu T, Li J, Yan M, Liu L, Lin H, Zhao F, Sun L, Zhang Y, Cui Y, Zhang F, et al: MicroRNA-193a-3p and -5p suppress the metastasis of human non-small-cell lung cancer by downregulating the ERBB4/PIK3R3/mTOR/S6K2 signaling pathway. Oncogene 34: 413-423, 2015

7. Ivashchenko A, Berillo O, Pyrkova A and Niyazova R: Binding sites of miR-1273 family on the mRNA of target genes. Biomed Res Int 2014: 620530, 2014.

8. Guo J, Sang Y, Yin T, Wang B, Yang W, Li X, Li H and Kang Y: miR-1273g-3p participates in acute glucose fluctuation-induced autophagy, dysfunction, and proliferation attenuation in human umbilical vein endothelial cells. Am J Physiol Endocrinol Metab 310: E734-E743, 2016.

9. Niu X, Fu N, Du J, Wang R, Wang Y, Zhao S, Du H, Wang B, Zhang Y, Sun D and Nan Y: miR-1273g-3p modulates activation and apoptosis of hepatic stellate cells by directly targeting PTEN in HCV-related liver fibrosis. FEBS Lett 590: 2709-2724, 2016.

10. Hou L, Su X, Qin X, et al: MiR-1273g-3p regulates A549 cell migration by targeting CNR1. J Med Mol Biol 11: $125-131,2014$

11. Pisanti S, Picardi P, D'Alessandro A, Laezza C and Bifulco M: The endocannabinoid signaling system in cancer. Trends Pharmacol Sci 34: 273-282, 2013.

12. Freundt-Revilla J, Kegler K, Baumgärtner W and Tipold A: Spatial distribution of cannabinoid receptor type 1 (CB1) in normal canine central and peripheral nervous system. PLoS One 12: e0181064, 2017.

13. Marshall AD, Lagutina I and Grosveld GC: PAX3-FOXO1 induces cannabinoid receptor 1 to enhance cell invasion and metastasis. Cancer Res 71: 7471-7480, 2011.

14. Guzmán M, Sánchez C and Galve-Roperh I: Control of the cell survival/death decision by cannabinoids. J Mol Med (Berl) 78: 613-625, 2001

15. Sánchez MG, Ruiz-Llorente L, Sánchez AM and Díaz-Laviada I: Activation of phosphoinositide 3-kinase/PKB pathway by $\mathrm{CB}(1)$ and $\mathrm{CB}(2)$ cannabinoid receptors expressed in prostate $\mathrm{PC}-3$ cells. Involvement in Raf-1 stimulation and NGF induction. Cell Signal 15: 851-859, 2003.

16. Livak KJ and Schmittgen TD: Analysis of relative gene expression data using real-time quantitative PCR and the 2(-Delta Delta C(T)) method. Methods 25: 402-408, 2001

17. Guindon $\mathrm{J}$ and Hohmann AG: The endocannabinoid system and cancer: Therapeutic implication. Br J Pharmacol 163: 1447-1463, 2011.
18. Greenhough A, Patsos HA, Williams AC and Paraskeva C: The cannabinoid delta(9)-tetrahydrocannabinol inhibits RAS-MAPK and PI3K-AKT survival signalling and induces BAD-mediated apoptosis in colorectal cancer cells. Int J Cancer 121: 2172-2180, 2007.

19. Jafri MA, Al-Qahtani MH and Shay JW: Role of miRNAs in human cancer metastasis: Implications for therapeutic intervention. Semin Cancer Biol 44: 117-131, 2017.

20. Huang LX, Hu CY, Jing L, Wang MC, Xu M, Wang J, Wang Y, Nan KJ and Wang SH: microRNA-219-5p inhibits epithelial-mesenchymal transition and metastasis of colorectal cancer by targeting lymphoid enhancer-binding factor 1 . Cancer Sci 108: 1985-1995, 2017.

21. Pisanti S, Malfitano AM, Grimaldi C, Santoro A, Gazzerro P, Laezza $\mathrm{C}$ and Bifulco M: Use of cannabinoid receptor agonists in cancer therapy as palliative and curative agents. Best Pract Res Clin Endocrinol Metab 23: 117-131, 2009.

22. Proto MC, Gazzerro P, Di Croce L, Santoro A, Malfitano AM, Pisanti S, Laezza $C$ and Bifulco $M$ : Interaction of endocannabinoid system and steroid hormones in the control of colon cancer cell growth. J Cell Physiol 227: 250-258, 2012.

23. Song $\mathrm{ZH}$ and Zhong M: CB1 cannabinoid receptor-mediated cell migration. J Pharmacol Exp Ther 294: 204-209, 2000.

24. Refolo MG, D'Alessandro R, Malerba N, Laezza C, Bifulco M, Messa C, Caruso MG, Notarnicola M and Tutino V: Anti proliferative and pro apoptotic effects of flavonoid quercetin are mediated by CB1 receptor in human colon cancer cell lines. J Cell Physiol 230: 2973-2980, 2015.

25. Neptune ER and Bourne HR: Receptors induce chemotaxis by releasing the betagamma subunit of $\mathrm{Gi}$, not by activating Gq or Gs. Proc Natl Acad Sci USA 94: 14489-14494, 1997.

26. Arai H, Tsou CL and Charo IF: Chemotaxis in a lymphocyte cell line transfected with $\mathrm{C}-\mathrm{C}$ chemokine receptor 2B: Evidence that directed migration is mediated by betagamma dimers released by activation of Galphai-coupled receptors. Proc Natl Acad Sci USA 94: 14495-14499, 1997.

27. Howlett AC, Qualy JM and Khachatrian LL: Involvement of Gi in the inhibition of adenylate cyclase by cannabimimetic drugs. Mol Pharmacol 29: 307-313, 1986.

28. Felder CC, Veluz JS, Williams HL, Briley EM and Matsuda LA: Cannabinoid agonists stimulate both receptor- and non-receptor-mediated signal transduction pathways in cells transfected with and expressing cannabinoid receptor clones. Mol Pharmacol 42: 838-845, 1992

29. Chen Y, Bai X, Zhang Q, Wen L, Su W, Fu Q, Sun X, Lou Y, Yang J, Zhang J, et al: The hepatitis B virus X protein promotes pancreatic cancer through modulation of the PI3K/AKT signaling pathway. Cancer Lett 380: 98-105, 2016

30. Karlsson E, Pérez-Tenorio G, Amin R, Bostner J, Skoog L, Fornander T, Sgroi DC, Nordenskjöld B, Hallbeck AL and Stål O: The mTOR effectors 4EBP1 and S6K2 are frequently coexpressed, and associated with a poor prognosis and endocrine resistance in breast cancer: A retrospective study including patients from the randomised Stockholm tamoxifen trials. Breast Cancer Res 15: R96, 2013.

31. Cai JQ, Xu XW, Mou YP, Chen K, Pan Y and Wu D: Upregulation of HOXB7 promotes the tumorigenesis and progression of gastric cancer and correlates with clinical characteristics. Tumour Biol 37: 1641-1650, 2016.

32. Wander SA, Zhao D, Besser AH, Hong F, Wei J, Ince TA, Milikowski C, Bishopric NH, Minn AJ, Creighton CJ and Slingerland JM: PI3K/mTOR inhibition can impair tumor invasion and metastasis in vivo despite a lack of antiproliferative action in vitro: Implications for targeted therapy. Breast Cancer Res Treat 138: 369-381, 2013. 